\title{
Mid- and high-J CO observations towards ultracompact HII regions
}

\author{
F. Wyrowski, S. Heyminck, R. Güsten, and K. M. Menten
}

Max-Planck-Institut für Radioastronomie, Auf dem Hügel 69, 53121 Bonn, Germany
e-mail: wyrowski@mpifr-bonn.mpg.de

Received 8 May 2006 / Accepted 18 May 2006

\section{ABSTRACT}

\begin{abstract}
Aims. A study of 12 ultracompact HII regions was conducted to probe the physical conditions and kinematics in the inner envelopes of the molecular clumps harboring them.

Methods. The APEX telescope was used to observe the sources in the $\mathrm{CO}(4-3)$ and ${ }^{13} \mathrm{CO}(8-7)$ lines. Line intensities were modeled with the RATRAN radiative transfer code using power laws for the density and temperature to describe the physical structure of the clumps.

Results. All sources were detected in both lines. The optically thick CO (4-3) line shows predominantly blue skewed profiles reminiscent of infall.

Conclusions. Line intensities can be reproduced well using the physical structure of the clumps taken from the literature. The optically thick line profiles show that $\mathrm{CO}$ is a sensitive tracer of ongoing infall in the outer envelopes of clumps harboring ultracompact HII regions and hot molecular cores.
\end{abstract}

Key words. stars: formation - ISM: clouds - ISM: kinematics and dynamics - ISM: structure - radio lines: ISM - submillimeter

\section{Introduction}

The earliest phases of massive star formation are still poorly understood. We know that massive stars are being born in dense clumps within giant molecular cloud complexes. Ultracompact HII regions (UCHIIRs) embedded within these clumps represent a key phase in the early lives of massive stars (see review by Hoare et al. 2005). UCHIIRs were defined by Wood \& Churchwell (1989) to have sizes $\leq 0.1 \mathrm{pc}$, densities $\geq 10^{4} \mathrm{~cm}^{-3}$, and emission measures $\geq 10^{7} \mathrm{pc} \mathrm{cm}^{-6}$. In their environs, often hot $(T>100 \mathrm{~K})$, compact $(<0.1 \mathrm{pc})$, and dense $\left(n\left(\mathrm{H}_{2}\right)>10^{7} \mathrm{~cm}^{-3}\right)$ cores are found, some of which are believed to be in a stage prior to the formation of UCHIIRs (Kurtz et al. 2000).

A better understanding of these clumps is a crucial prerequisite for models of high-mass star formation. Several studies have attacked this topic in the past. In particular, Hofner et al. (2000) conducted a $\mathrm{C}^{17} \mathrm{O}$ survey of 16 UCHIIRs and found typical sizes of $1 \mathrm{pc}$ for the clumps and average densities and temperatures of $10^{5} \mathrm{~cm}^{-3}$ and $25 \mathrm{~K}$. These values, obtained under simple assumptions, are an important first approximation but the high luminosities and densities of the embedded hot cores show that a proper representation of the physical conditions of the clumps requires density and temperature gradients. Hatchell \& van der Tak (2003) present models with power laws for the density and temperatures that were constrained by the spectral energy distributions (SEDs) of the sources and single-dish continuum images and CS line data. One drawback of using CS is the abundance variation of this molecule with changing physical environments. Toward the sources studied by Hatchell \& van der Tak (2003), the abundance varies by almost three orders of magnitudes which suggests that within an individual envelope the abundance might also vary considerably. Therefore, in the inner regions of the clumps, mid- and high- $J$ transitions of $\mathrm{CO}$ with a stable abundance in warm regions are better probes of the physical conditions.

One of the important results in the study of low-mass star formation has been the observation of infall motions (e.g. Belloche et al. 2002), which give direct evidence of accretion. Toward high-mass star-forming cores, the observational evidence of infall is still very scarce (e.g. Wu \& Evans 2004). In addition to probing density and temperature, mid- and high- $J$ lines of $\mathrm{CO}$ allow the kinematics of the cores to be probed, hence to search for further evidence of infall.

Here, we present a study of 12 UCHIIRs, mostly with associated hot cores, in the $\mathrm{CO}(4-3)$ and ${ }^{13} \mathrm{CO}(8-7)$ lines to probe the physical conditions and kinematics of the inner envelopes of the clumps harboring the UCHIIRs.

\section{Observations}

The observations were made with the Atacama Pathfinder Experiment (APEX ${ }^{1}$, Güsten et al. 2006) in June 2005. The frontend used was the MPIfR dual channel (460 and $810 \mathrm{GHz}$ ) FLASH receiver (Heyminck et al. 2006), which enables simultaneous observations of the ${ }^{12} \mathrm{CO}(4-3)$ and ${ }^{13} \mathrm{CO}(8-7)$ lines. As backends, the MPIfR Fast Fourier Transform Spectrometers (FFTS, Klein et al. 2006) were used for the line observations. The lines were covered with 2048 channels within a bandwidth of $1 \mathrm{GHz}$, resulting in spectral resolutions of 0.32 and $0.17 \mathrm{~km} \mathrm{~s}^{-1}$. The spectra were converted from $T_{\mathrm{A}}^{*}$ to $T_{\mathrm{MB}}$ units using forward efficiencies of 0.95 and beam efficiencies of 0.6 and 0.43 at 461 and $881 \mathrm{GHz}$, respectively.

1 This publication is based on data acquired with the Atacama Pathfinder Experiment (APEX). APEX is a collaboration between the Max-Planck-Institut für Radioastronomie, the European Southern Observatory, and the Onsala Space Observatory. 

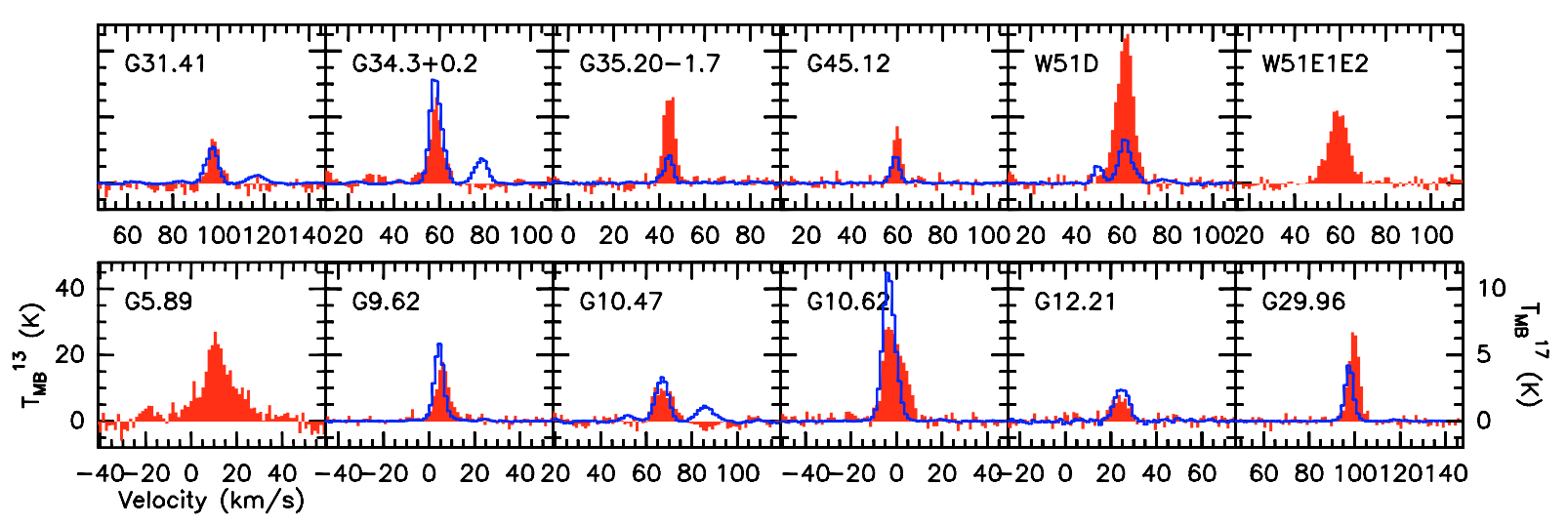

Fig. 1. APEX ${ }^{13} \mathrm{CO}(8-7)$ spectra (filled histograms) compared with $\mathrm{C}^{17} \mathrm{O}(2-1)$ observations (blue line, from Hofner et al. 2000). The spectral features appearing at -15 and $19 \mathrm{~km} \mathrm{~s}^{-1}$ in some of the $\mathrm{C}^{17} \mathrm{O}$ spectra are from other molecules.

Table 1. Source list.

\begin{tabular}{lcc}
\hline \hline Source & RA (J2000) & Dec (J2000) \\
\hline G5.89-0.39 & $18: 00: 30.376$ & $-24: 04: 00.48$ \\
G9.62+0.19 & $18: 06: 15.000$ & $-20: 31: 42.10$ \\
G10.47+0.03 & $18: 08: 38.218$ & $-19: 51: 49.71$ \\
G10.62-0.38 & $18: 10: 28.661$ & $-19: 55: 49.77$ \\
G12.21-0.10 & $18: 12: 39.700$ & $-18: 24: 20.00$ \\
G29.96-0.02 & $18: 46: 03.950$ & $-02: 39: 21.40$ \\
G31.41+0.31 & $18: 47: 34.401$ & $-01: 12: 45.95$ \\
G34.26+0.15 & $18: 53: 18.499$ & $01: 14: 58.66$ \\
G35.20-1.74 & $19: 01: 46.440$ & $01: 13: 23.50$ \\
W51D & $19: 23: 39.946$ & $14: 31: 08.13$ \\
W51E1E2 & $19: 23: 43.762$ & $14: 30: 26.40$ \\
G45.12+0.13 & $19: 13: 27.808$ & $10: 53: 36.72$ \\
\hline
\end{tabular}

The CO (4-3) line was observed for several sources on two different days and the observed line temperatures agree within $20 \%$. The beam sizes at the observing frequencies are 13 and $7^{\prime \prime}$. All observations were done in position switching mode on the UCHIIR positions given in Table 1 using offpositions $250^{\prime \prime}$ to the east. These sources were selected from the sample studied by Hofner et al. (2000) with the addition of the two sources in W51. Pointing was done regularly on strong submm continuum sources in the sample (G10.47 and G34.26) and should be accurate within $2^{\prime \prime}$.

\section{Results}

Figure 1 shows the observed ${ }^{13} \mathrm{CO}(8-7)$ spectra compared with $\mathrm{C}^{17} \mathrm{O}(2-1)$ spectra from Hofner et al. (2000) and line parameters from Gaussian fits to the spectra are given in Table 2. All sources are clearly detected and show line shapes similar to $\mathrm{C}^{17} \mathrm{O}$. Notable deviation from Gaussian shapes are seen in G10.62, with a strong red wing, and in G5.89-0.39, where the high velocity outflow wings are prominent. The ${ }^{13} \mathrm{CO}$ linewidths are in all cases typically larger by $2 \mathrm{~km} \mathrm{~s}^{-1}$. While line broadening can be caused by high optical depths, the absence of complicated self-absorbed spectral shapes suggests rather moderate optical depths, hence a true increase in motions. For most sources, the ratio of the ${ }^{13} \mathrm{CO}(8-7)$ and $\mathrm{C}^{17} \mathrm{O}(2-1)$ line temperatures is the same within a factor 2, with G35.20-1.7 and W51D being the exceptions with much stronger ${ }^{13} \mathrm{CO}$ lines.

The observed ${ }^{12} \mathrm{CO}(4-3)$ spectra are shown in Fig. 2 compared with CS (7-6) spectra from Olmi et al. (1999). All sources show complicated line profiles with self-absorption and outflow wings. The self-absorption is in most cases redshifted compared
Table 2. APEX ${ }^{13} \mathrm{CO}(8-7)$ line parameters.

\begin{tabular}{lccc}
\hline \hline Source & $\begin{array}{c}T_{\mathrm{MB}} \\
(\mathrm{K})\end{array}$ & $\begin{array}{c}v_{\mathrm{LSR}} \\
\left(\mathrm{km} \mathrm{s}^{-1}\right)\end{array}$ & $\begin{array}{c}F W H M \\
\left(\mathrm{~km} \mathrm{~s}^{-1}\right)\end{array}$ \\
\hline G5.89 & $20.8(2.1)$ & $12.0(0.4)$ & $20.9(1.2)$ \\
G9.62 & $16.3(1.0)$ & $6.2(0.1)$ & $7.0(0.3)$ \\
G10.47 & $11.5(1.9)$ & $67.7(0.5)$ & $12.4(1.1)$ \\
G10.62 & $27.7(1.3)$ & $-1.1(0.1)$ & $10.8(0.3)$ \\
G12.21 & $6.9(1.5)$ & $23.9(0.5)$ & $9.7(1.2)$ \\
G29.96 & $26.9(1.0)$ & $99.9(0.1)$ & $5.6(0.2)$ \\
G31.41 & $14.0(1.3)$ & $98.3(0.2)$ & $6.6(0.5)$ \\
G34.26 & $23.1(2.1)$ & $58.8(0.2)$ & $7.2(0.6)$ \\
G35.20 & $27.3(1.3)$ & $44.6(0.1)$ & $6.0(0.2)$ \\
W51D & $43.9(1.7)$ & $61.6(0.1)$ & $8.7(0.2)$ \\
W51E1E2 & $21.5(1.3)$ & $59.4(0.2)$ & $10.2(0.4)$ \\
G45.12 & $17.3(1.3)$ & $60.2(0.1)$ & $4.1(0.3)$ \\
\hline
\end{tabular}

to the velocity of the optically thin $\mathrm{C}^{17} \mathrm{O}$ lines, with only G9.62 and G35.20 showing a blueshifted self-absorption. The line shapes are either double peaked with a stronger blue peak or skewed to the blue. G9.62 is again an exception and G45.12 shows a rather symmetric profile. G5.89 is dominated by the strong outflow and absorbing foreground clouds (Klaassen et al. 2006). Also in the red line wing of G9.62, absorption due to foreground clouds is seen, consistent with the $\mathrm{HCO}^{+}$observations by Hofner et al. (2001). The asymmetries in the line shapes of the CS (7-6) line are mostly consistent with those we find in ${ }^{12} \mathrm{CO}(4-3)$ but the self absorption is weaker. To quantify the asymmetries seen in the profiles to check for infall signatures (Table 3), we determined the ratio of blue-to-red peak intensity for the 8 sources with 2 peaks. Seven sources show significantly stronger blue peaks, and the average ratio of the sample is 3.3 , thus indicative of infall. For the remaining 3 sources with only one peak (G5.89 was omitted due to its strong outflow), we determined $\delta v=\left(v_{\text {thick }}-v_{\text {thin }}\right) / \Delta v_{\text {thin }}$, the difference between optically thick $\left({ }^{12} \mathrm{CO}\right)$ and thin $\left(\mathrm{C}^{17} \mathrm{O}\right)$ line peaks over the optically thin line widths, which can be used as an infall indicator (Mardones et al. 1997). For 2 sources $\delta v$ is -0.5 , hence clearly blue-shifted, and for 1 it is close to 0 .

\section{Discussion}

The CO emission of the sources has been modeled with the Monte Carlo radiative transfer program of Hogerheijde \& van der Tak (2000). Besides collisional excitation, dust radiation is taken into account using grain properties from Ossenkopf \& Henning (1994), Model 5. The radial profile of the density, 


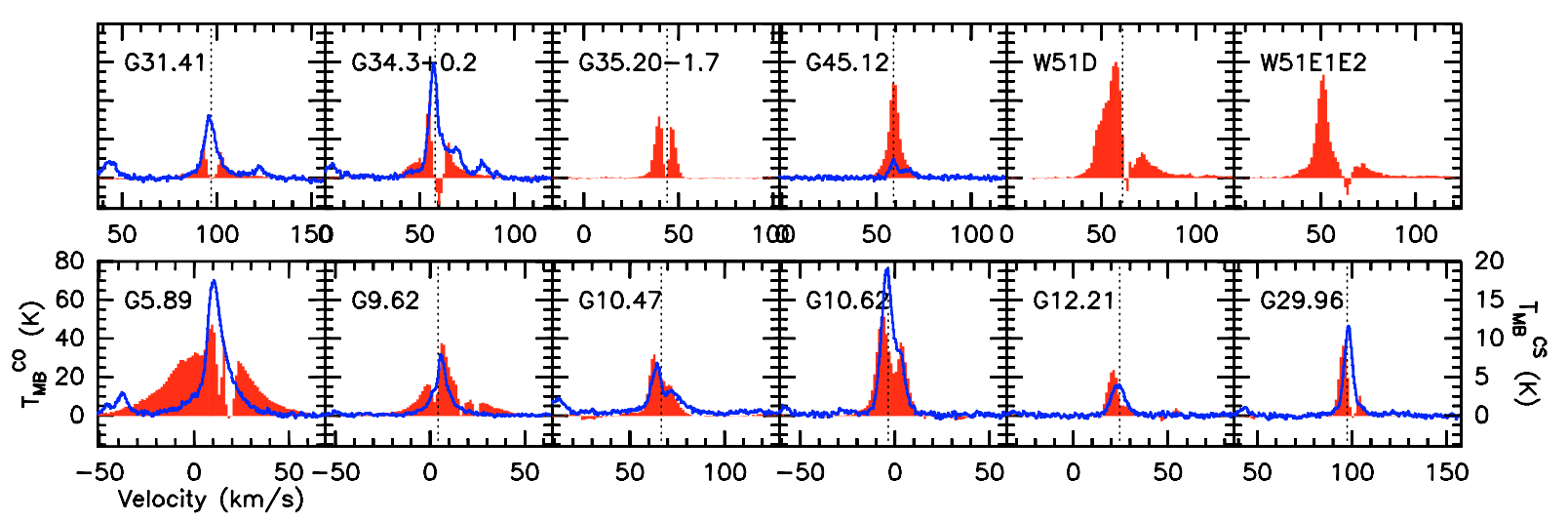

Fig. 2. APEX CO (4-3) spectra (filled histograms) compared with CS (7-6) observations from Olmi \& Cesaroni (1999). The velocity from $\mathrm{C}^{17} \mathrm{O}$ is marked with a dashed line.

Table 3. APEX ${ }^{12} \mathrm{CO}(4-3)$ collapse indicators. For two-peaked profiles the ratio of blue and red peak and for the single-peaked lines the skewness parameter $\delta v$ are given. The profile column denotes blue $(B)$ and red $(R)$ line profiles.

\begin{tabular}{lccc}
\hline \hline Source & $T_{\text {blue }} / T_{\text {red }}$ & $\delta v$ & Profile \\
\hline G9.62 & 0.44 & & $R$ \\
G10.47 & & -0.50 & $B$ \\
G10.62 & 1.38 & & $B$ \\
G12.21 & & -0.51 & $B$ \\
G29.96 & 4.21 & & $B$ \\
G31.41 & 1.40 & & $B$ \\
G34.26 & 2.03 & & $B$ \\
G35.20 & 1.42 & & $B$ \\
W51D & 4.67 & & $B$ \\
W51E1E2 & 7.87 & & $B$ \\
G45.12 & & 0.04 & \\
\hline
\end{tabular}

as well as inner and outer radii, were taken from the best-fit DUSTY models of Hatchell \& van der Tak (2003, RATRAN code) based on single-dish maps of dust continuum and CS molecular line emission. They determined power laws for the density structure with power law exponents between -1.5 and -2.0 for our sample. RATRAN and DUSTY were used with the same dust properties. The temperature structure was then solved by DUSTY (Ivezić et al. 1997) in a self-consistent way. An isotopic ratio of 40 and 2000 was used for ${ }^{13} \mathrm{CO}$ and $\mathrm{C}^{17} \mathrm{O}$, respectively, relative to ${ }^{12} \mathrm{CO}$. To simplify the modeling, no variable velocity field was used but only constant, turbulent line widths. This is a reasonable approximation of the integrated intensities of lines that are optically thin or have only moderate optical depths; but for optically thick lines like the ${ }^{12} \mathrm{CO}(4-3)$ line, the resulting integrated intensities will depend crucially on the assumed velocity fields. Therefore, in Fig. 3 only the integrated ${ }^{13} \mathrm{CO}(8-7)$ and $\mathrm{C}^{17} \mathrm{O}(2-1)$ line intensities from the observations and the modeling are compared. For this comparison, the modeled intensities were determined from the RATRAN output images by convolving with the APEX and $30 \mathrm{~m}$ observing beams. The agreement between the modeled intensities using the Hatchell \& van der Tak fit results and the observations is remarkable. The only large deviations are the predictions of the $\mathrm{C}^{17} \mathrm{O}$ intensities for G10.47 and G31.41 for which the outer radius of the clumps might be overestimated, and this mostly affects the $\mathrm{C}^{17} \mathrm{O}(2-1)$ lines and not ${ }^{13} \mathrm{CO}(8-7)$. Since the modeled lines probe very different excitation regimes and are completely independent of the data used by Hatchell \& van der Tak, this

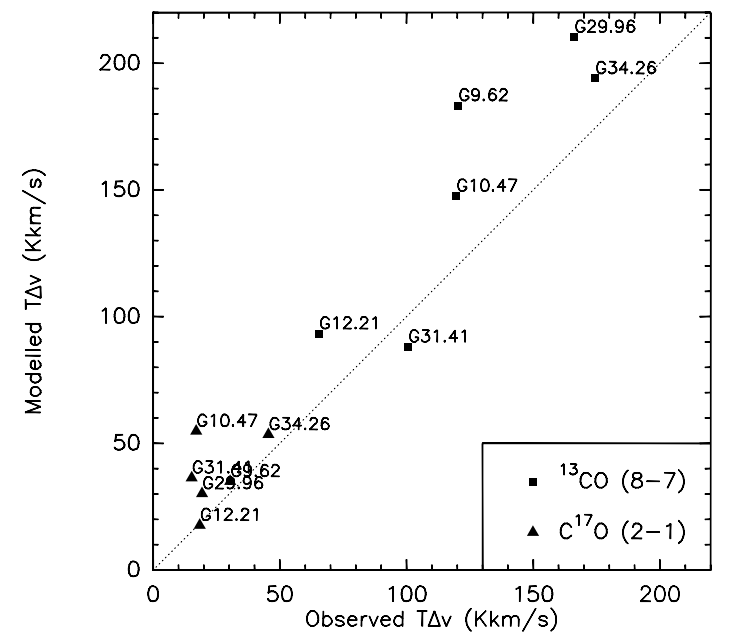

Fig. 3. Comparison between modeled and observed integrated $\mathrm{C}^{17} \mathrm{O}$ and ${ }^{13} \mathrm{CO}$ intensities. The modeled intensities were obtained using the physical structure of the clumps determined by Hatchell \& van der Tak (2003).

agreement is a strong validation of the power law structure in $T$ and $n$ of the clumps.

The observed ${ }^{12} \mathrm{CO}$ profiles are very reminiscent of infall asymmetries routinely detected toward low-mass star-forming cores (e.g. Myers et al. 2000). For individual sources, there is still the possibility that some of the absorption might be caused by colder foreground clouds, but this would not explain the general trend of redshifted absorption and most of the blue peaks being stronger. Also, in several cases the observed infall signature is consistent with results from interferometric observations where the infall is observed mostly in absorption using different molecules. Examples are G10.62 $\left(\mathrm{NH}_{3}\right.$, Keto et al. 1988), W51E $\left(\mathrm{HCO}^{+}\right.$, Rudolph et al. 1990) and G29.96 $\left(\mathrm{HCO}^{+}\right.$, Maxia et al. 2001). We can infer a total of 9 out of 11 sources with infall evidence from the line profiles discussed in Sect. 3 . The excess parameter $E=\left(N_{\text {blue }}-N_{\text {red }}\right) / N_{\text {tot }}$, introduced by Mardones et al. (1997) to quantify the statistics of infall surveys, is for our sample 0.7 , which is larger than for low-mass samples or the massive star-forming region survey by Wu \& Evans (2003). This might be a selection effect, since mostly UCHIIRs with associated hot cores, hence early stages of massive star formation, were targeted. It might also be related to using $\mathrm{CO}$ as an infall tracer. The excess parameter using CS for our sample would have been smaller. The high optical depths of the 
CO (4-3) line make it a very sensitive probe of infalling motions in the outer envelopes of the massive star-forming clumps. Therefore, while observations of much higher- $J$ optically thick $\mathrm{CO}$ lines are needed to probe the kinematics in the inner part of the clumps, the $\mathrm{CO}(4-3)$ observations clearly show that in the outer parts (a remnant) infall is still going on.

In the near future, the upcoming CHAMP+ array - a $2 \times$ 7 pixel heterodyne array for simultaneous observations in the 350 and $450 \mu \mathrm{m}$ atmospheric windows to be installed at the APEX telescope in the middle of 2006 - will allow us to image high- $J$ CO lines and then, together with proper models of the density and temperature structure, put further and tighter constraints on the velocity structure of the massive star-forming clumps.

Acknowledgements. We thank Riccardo Cesaroni for providing the CS (7-6) spectra in CLASS format.

\section{References}

Belloche, A., André, P., Despois, D., \& Blinder, S. 2002, A\&A, 393, 927

Hatchell, J., \& van der Tak, F. F. S. 2003, A\&A, 409, 589

Güsten, R., Nyman, L. Å., Schilke, P., et al. 2006, A\&A, 454, L13
Heyminck, S., Kasemann, C., G’usten, R., de Lange, G.,\& Graf, U. U. 2006, A\&A, 454, L21

Hoare, M. G., Kurtz, S. E., Lizano, S., Keto, E., \& Hofner, P. 2006, to appear in Protostars and Planets V, ed. B. Reipurth, D. Jewitt, \& K. Keil (Tucson: University of Arizona Press)

Hofner, P., Wyrowski, F., Walmsley, C. M., \& Churchwell, E. 2000, ApJ, 536, 393

Hofner, P., Wiesemeyer, H., \& Henning, T. 2001, ApJ, 549, 425

Hogerheijde, M. R., \& van der Tak, F. F. S. 2000, A\&A, 362, 697

Ivezić, Z., Nenkova, M., \& Elitzur, M. 1999, User Manual for Dusty, University of Kentucky Internal Report, accessible at http: //www. pa.uky. edu/ moshe/dusty

Keto, E. R., Ho, P. T. P., \& Haschick, A. D. 1988, ApJ, 324, 920

Klaassen, P. D., Plume, R., Ouyed, R., von Benda-Beckmann, A. M., \& Di Francesco, J. 2006, ApJ, in press

Klein, B., Philipp, S. D., Kr'amer, I., et al. 2006, A\&A, 454, L29

Kurtz, S., Cesaroni, R., Churchwell, E., Hofner, P., \& Walmsley, C. M. 2000, Protostars and Planets IV, 299

Mardones, D., Myers, P. C., Tafalla, M., et al. 1997, ApJ, 489, 719

Maxia, C., Testi, L., Cesaroni, R., \& Walmsley, C. M. 2001, A\&A, 371, 287

Myers, P. C., Evans, N. J., \& Ohashi, N. 2000, Protostars and Planets IV, 217

Olmi, L., \& Cesaroni, R. 1999, A\&A, 352, 266

Ossenkopf, V., \& Henning, T. 1994, A\&A, 291, 943

Rudolph, A., Welch, W. J., Palmer, P., \& Dubrulle, B. 1990, ApJ, 363, 528

Wood, D. O. S., \& Churchwell, E. 1989, ApJS, 69, 831

Wu, J., \& Evans, N. J. 2003, ApJ, 592, L79 